\title{
Efe Çaman \\ Türkei quo vadis? \\ Determinanten türkischer Außenpolitik in Europa und Asien
}

Die Türkei als Staat zwischen Europa, dem Nahen Osten und dem Kaukasus oder wie die türkischen Führungseliten ihr Land als eine Brücke zwischen Asien und Europa sowie den muslimischen und abendländischen Kulturen wahrnehmen, ${ }^{1}$ war und ist ein wichtiger Akteur in der internationalen Politik. In der Zeitperiode seit dem Zerfall der Sowjetunion fanden bedeutende Änderungen in den oben genannten Regionen statt. Während die ehemalig sozialistischen Staaten Osteuropas in dieser Zeitperiode versucht haben, eine gelungene Integration in die Europäische Union (EU) zu verwirklichen und ihr politisches und ökonomisches System entsprechend den Aufnahmekriterien der EU zu transformieren, sind es nun seit der Auflösung der Sowjetunion im postsowjetischen Norden und Osten der Türkei neue, ehemals sowjetische, seit Beginn der 90er Jahren unabhängige Staaten, die in der neu entstandenen Konstellation ihre Eigenstaatlichkeit und ihre eigenen politischen Wege behaupten.

Die Türkei befindet sich aufgrund ihrer geographischen Lage in der Schnittstelle zwischen diesen Regionen und mehr als das: sie ist Teil von ihnen. Deshalb ist sie ständiger Beeinflussung durch die politischen Konstellationen in oben angesprochenen Regionen, vor allem aber - aufgrund seines Gewichtes in der türkischen Außenpolitik - durch Europa unterworfen. Die Veränderungen im regionalen Umfeld übten Einfluss auf die Außen, Innen- und Sicherheitspolitik des Landes aus, auf die ökonomische Entwicklung sowie auf die politischen Perzeptionen seiner Eliten. Es ist ein Faktum, dass die Konstanten bzw. Bestimmungsfaktoren der türkischen Außenpolitik, die seit dem Ende des Zweiten Weltkriegs bis zum Ende des bipolaren internationalen Systems bestanden haben, nach dem Ende des Ost-West-Konflikts ihre Gültigkeit größtenteils verloren haben, und die Beziehungen der Türkei zu den oben erwähnten Regionen und zu ihren einzelnen Akteuren - vor allem in Europa und im postsowjetischen Osten - seit dem Ende des Kalten Kriegs neu geordnet werden.

In dieser Abhandlung wird die türkische Außenpolitik nach dem Ende des OstWest-Konflikts behandelt. Herausgearbeitet und dargestellt werden Teilbereiche der türkischen Außenpolitik. Vor allem wird versucht, die grundlegende Frage zu

1 Insbesondere seit dem Amtseintritt der muslim-demokratischen AKP-Regierung wird im außenpolitischen Kontext diese Funktion des Landes betont in Vordergrund gestellt.

ZfP 53.Jg. 4/2006 
beantworten, nämlich ob die türkischen Entscheidungsträger mit ihrer neuen Regionalpolitik nach dem Ende des Ost-West-Konflikts eine fundamentale Neuorientierung als Alternative zu ihrer traditionellen Europaorientierung zu verwirklichen beabsichtigten und ob eine solche Chance in Wirklichkeit bestand. Ausgehend von dieser leitenden Frage wird versucht, die neue türkische Außenpolitik nach dem Ende des Ost-West-Konflikts zu verorten.

\section{Exogene Determinanten der Außenpolitik}

Die neunziger Jahre könnten für die türkische Außenpolitik in vielerlei Hinsicht als eine prekäre Zeitperiode bezeichnet werden. Die Desintegration der Sowjetunion und die daraus folgenden Erschütterungen in der regionalen Umgebung der Türkei, die sich meistens in Form von neuen Instabilitäten, Konfliktpotenzialen und Konflikten sowie (außen)politischen und sicherheitspolitischen Unwägbarkeiten manifestierten, beunruhigten die türkischen Entscheidungsträger und Führungseliten vom Ende des Ost-West-Konflikts an zunehmend, da sie entsprechend der republikanischen Tradition an der Erhaltung des regionalen Status-quo auf Grundlage des Friedensvertrages von Lausanne ${ }^{2}$ interessiert waren, der nach dem Ende des Ersten Weltkrieges nicht nur die innenpolitischen Parameter des Landes, sondern auch die außenpolitischen Reflexe und Denkweisen bestimmend beeinflusste. Das Ende des Ost-West-Konflikts brachte für das regionale Umfeld der Türkei jedoch alles andere als eine Garantie der Bewahrung des Status-quo.

Wie die Führungseliten in anderen Ländern der Region, vor allem aber in Europa, waren auch die türkischen Entscheidungsträger von der unerwartet entstandenen neuen Konstellation nach Ende des Ost-West-Konflikts überrascht. Die neue regionale Konstellation führte erstens zu Veränderungen der Rahmenbedingungen der bisherigen Außenpolitik - dies impliziert vor allem die kontinuierliche Europapolitik des Landes mit dem Endziel des EU-Beitritts - und zweitens zur Gestaltung einer neuen regional ausgerichteten Außenpolitik.

Es ist im Hinblick auf eine ganzheitliche Korrelierung und Bewertung von großer Bedeutung, die neue regionale Konstellation mit ihren Rahmenbedingungen im Zusammenhang der türkischen Außenpolitik zu thematisieren. Das in dem Desintegrationsprozess der Sowjetunion entstandene Vakuum jenseits der nordöstlichen Grenzen (türkisch-sowjetische Grenze) des Landes brachte neue exogene Einflussfaktoren hervor, die im Prozess des Policy-Making von den türkischen Entscheidungsträgern unbedingt mit berücksichtigt werden mussten. Dabei waren sie allerdings überfordert, zumal das bestehende politische Vakuum alle Komponenten der internationalen Konstellation permanent beeinflusste, d.h. es herrschte nach der Desintegration des Ostblocks intensive politische Wandlungsprozesse. Insbesonde-

2 Für die Bestimmungen des Friedensvertrages von Lausanne siehe Adnan San, Die Stellung der Türkei im Rabmen internationaler Verträge seit dem Ersten Weltkrieg, Göttingen 1963, S. $18 f$. 
re auf dem europäischen Kontinent sind neue Verhältnisse entstanden, die gewichtige Auswirkungen auf die politischen Entwicklungen in Westeuropa hatten. Vor allem realisierten die Führungseliten der EU eine neue, während des Ost-WestKonflikts undenkbar gewesene Dimension der europäischen Integration. Die EU wurde zu einem Gravitationszentrum für Osteuropa und setzte sich das ehrgeizige und historische Ziel, ein politisch, wirtschaftlich und sicherheitspolitisch vereinigtes Europa zu verwirklichen, das es in der europäischen Geschichte bisher nicht gab. Seitdem befinden sich die ehemals sozialistischen osteuropäischen Staaten des Warschauer Paktes sowie die drei postsowjetischen baltischen Länder unter dem dominierenden Einfluss einer alternativlosen Systemtransformation und in einem Integrationsprozess mit der EU.

Das nach der Auflösung der Sowjetunion entstandene Vakuum löste auch im Kaukasus und in Zentralasien gravierende Änderungen aus. Die Türkei wurde daher wie die anderen Staaten in der Region tiefgreifend von diesen internationalen Ereignissen beeinflusst. Alleine die Tatsache, dass sogar die gemeinsame türkischsowjetische Grenze durch die Desintegration der Sowjetunion verschwand und auf ehemals sowjetischem Territorium drei neue Staaten entstanden, mit denen die Türkei nun jeweils eine Grenze hatte, zeigt das Ausmaß der Veränderung für die türkische Außenpolitik. Die Entstehung der neuen postsowjetischen Nationalstaaten im Kaukasus und in Zentralasien war eine Entwicklung, die die türkischen Entscheidungsträger nicht ignorieren konnten.

Die neue Konstellation, deren Folgen oben zusammengefasst wurden, brachte in ihrer Anfangsphase aus der Perspektive der türkischen Außenpolitik simplifiziert ausgedrückt zwei Ergebnisse von besonderer Relevanz mit sich. Zum einen verursachte sie einen gewissen Verlust der sicherheitspolitischen Relevanz der Türkei im Hinblick auf die Interessen der westeuropäischen Staaten. ${ }^{3}$ Die Türkei rückte folglich in die äußerste Peripherie Europas, was die Haltung der EU-Staaten gegenüber der Türkei im Zusammenhang der türkischen Europaintegration nicht im Sinne der türkischen Interessen beeinflusste. Zum anderen veränderte die neue Konstellation die regionalen Konditionen und eröffnete neue außen-, kultur- und auch machtpolitische, aber auch außenwirtschaftliche Optionen für das Land.

Im Spannungsfeld zwischen diesen beiden Folgen des Endes des Ost-West-Konflikts waren türkische Führungseliten mit der oben erwähnten Problematik der außenpolitischen Orientierung einer nun erforderlich gewordenen außenpolitischen Neugestaltungsnotwendigkeit konfrontiert, die nicht nur eine außenpolitische Neubeurteilung der internatonalen Lage erforderte, sondern auch realistische Perzeptionen bezüglich der neuen Konstellation verlangte.

3 Şule Kut: »The Contours of Turkish Foreign Policy in the 1990s « in: Barry Rubin, / Kemal Kirişçi, Turkey in World Politics. An Emerging Multiregional Power, Istanbul 2002, S. 8. Siehe auch Kemal Kirişçi, »Uluslararası Sistemdeki Değişmeler ve Türk Diş Politikasının Yeni Yönelimleri« in: Faruk Sönmezoğlu (Hg.), Türk Dış Politikasının Analizi, Istanbul 1994, S. $393 \mathrm{ff}$. 


\section{Wandel der Perzeptionen}

Die Beziehungen Europas zur Türkei wurden während des Kalten Krieges ausschließlich aus geostrategischen und sicherheitspolitischen Blickwinkeln gerechtfertigt und begründet. Die Priorität, das westlich-freiheitliche Europa gegenüber der expansionistischen sowjetischen Bedrohung zu schützen, bekräftigte die Position des südöstlichen NATO-Partners infolge seiner Funktion in der europäischen Sicherheitsstruktur auch im Kontext der europäischen Integration.

Die Perspektive, an der europäischen Integration teilzunehmen, beinhaltete jedoch für die Türkei auch identitätsstiftende Funktionen. Die Eliten der Republik Türkei gründeten und definierten das Land ganz im Sinne der europäischen Aufklärung als einen europäischen Staat. Es handelte sich bei der Europa-Orientierung um eine Entscheidung der türkischen Führungseliten und Intellektuellen. Die These der sich unter den besonderen Konditionen des Kalten Kriegs europäisierenden Türken entspricht also nicht den historischen Tatsachen. ${ }^{4}$

Die Selbstperzeption der türkischen Eliten wurde von den europäischen Eliten vorwiegend nicht registriert. In Europa blieb die identitätsstiftende Rolle der Türken als das Andere - meistens als Gegenteil des Europäerseins - erhalten, während in der Türkei Europa als das Andere parallel zur zunehmenden Europäisierung und außenpolitischen Europaorientierung der Türken überwiegend relativiert wurde. Die türkische Selbstperzeption gab bei der Intensivierung der Beziehungen der Türkei zu Europa relevante Impulse und motivierte die Entscheidungsträger des Landes ununterbrochen dazu, an den institutionalisierten Kooperationen in Europa teilzunehmen.

Die neue Konstellation nach der Desintegration der Sowjetunion transformierte nicht nur die ehemals sozialistischen osteuropäischen Staaten, sondern auch die Perzeptionsweise westeuropäischer Staaten. Das europäische Integrationsprojekt war nach dem Ende des Ost-West-Konflikts die Trägerin gesamteuropäischer Erwartungen. ${ }^{5}$ Ironischerweise entstanden erst nach der Entstehung dieser gesamteuropäischen Perspektive die Tendenzen in Europa, den Platz der Türkei in Europa infrage zu stellen.

\section{Chance einer neuen Regionalpolitik}

Die neue Konstellation in der Region eröffnete neue außenpolitische Möglichkeiten für die Türkei. Sie erforderte eine neue Bewertung und Beurteilung der neuen Ausgangslage in der Regionalpolitik, in der nun auch endogene Determinanten, vor allem ideologischer Natur, zumindest bei den Perzeptionen türkischer Entschei-

4 Für die Einzelheiten dieser Position siehe z.B. Hans Arnold, Europa am Ende? Die Auflösung von EG und NATO, München 1993, S. 31.

5 Werner Weidenfeld, »Europa - aber wo liegt es? «, in: ders. (Hg.): Europa Handbuch, Bonn 1999, S. 19. 
dungsträger, Führungseliten und Intellektuellen eine Rolle zu spielen begannen. In diesem Kontext stellte es sich heraus, dass die vorrepublikanische Vergangenheit des Landes auch im Zusammenhang mit der Haltung der Staaten in den benachbarten Regionen - z.B. auf dem Balkan, im Schwarzmeerraum, im Kaukasus und im Mittleren Osten - bei der Gestaltung der Regionalpolitik von nicht zu unterschätzender Bedeutung war, obwohl die offizielle Geschichtsschreibung der Republik das historische und kulturelle Erbe ihres Vorgängers, des Osmanischen Imperiums, beinahe völlig ignorierte.

Das Ende des Ost-West-Konflikts beeinflusste die Perzeption der Führungseliten im Kindermannschen Sinne im Hinblick auf die Bewertung der Gegenwart und die Perzeption der Daseinslagen, auf die rückblickende Deutung geschichtlicher Entwicklungen sowie auf Zukunftstendenzen. Hierbei spielten das ideologische Dreieck, der Kemalismus, der Islamismus und der Nationalismus, als endogene Determinanten der türkischen Außenpolitik eine einflussreiche Rolle. Einerseits bilden die Anhänger dieser Ideologien ab dem Beginn der neunziger Jahre einen Teil der gesellschaftlichen Pluralität in der politischen Landschaft des Landes und trugen in diesem Sinne zur neu entstehenden offeneren und toleranteren politischen Kultur bei. Andererseits - und noch wichtiger - scheinen die Ideologien nach dem Ende des Ost-West-Konflikts auch im außenpolitischen Kontext sowohl im Hinblick auf die Bildung neuer Perzeptionen der türkischen Entscheidungsträger als auch dementsprechend im außenpolitischen Entscheidungsprozess des Landes wichtiger zu sein. Die selektiv-subjektive Bestandsaufnahme der türkischen Entscheidungsträger hängt auch mit den ideologischen Bestimmungsfaktoren zusammen.

Angesichts der gesellschaftlichen Pluralität verfügen die Ideologien in der Türkei nach dem Ende des Ost-West-Konflikts über genügend Nährboden für einen Evolutionsprozess. Die Transformation des Kemalismus und des Islamismus in Pluralform $^{6}$ ist ein Indiz dieser positiven Entwicklung. Die Spannung zwischen den laizistisch orientierten Kemalisten und den das laizistische System des Landes zu relativieren beabsichtigenden Islamisten manifestierte sich als eine relevante endogene Kodeterminante mit außenpolitisch nicht zu unterschätzenden Folgen. Während der sozialdemokratisch tendierte Kemalismus - vor allem der CHP - sich in neunziger Jahren nicht nur im innen- sondern auch außenpolitischen Kontext zunehmend $\mathrm{zu}$ einer Status-quo-Ideologie entwickelte, entfesselte sich der Islamismus der AKP zwar nicht völlig jedoch zunehmend von seinen fundamentalistischen Wurzeln. Im Kemalismus als staatstragender Ideologie konkretisiert sich die früher eher marginal interpretierbare Teilung in Staatskemalismus und alternative Kemalismen. Dennoch bleibt der staatstragende Kemalismus die dominante Version dieser Ideologie und wird insbesondere vom Militär und von der Mehrheit der Bürokratie sowie von den linksgerichteten Parteien (CHP und DSP) überwiegend konservativ interpretiert.

6 Dabei muss darauf hingewiesen werden, dass es mehrere Islamismen gibt und daher die Islamisten nicht als ein einheitlicher Block betrachtet werden sollten. Siehe hierzu Peter Antes, Der Islam als politischer Faktor, Bonn 1997, S. 92 f. 
Der Konservativismus im an sich reformistisch und radikal-modernistisch orientierten Kemalismus scheint paradox zu sein, aber es ist im Kontext des Staatslaizismuskonzepts eine Tatsache, der im Endeffekt die staatliche Kontrolle der Religion vorsieht, um deren Einfluss auf die Politik zu verhindern. In der Außenpolitik gewinnt das Gewicht des Kemalismus gerade in diesem innenpolitischen Kontext an Relevanz. Das Spannungsfeld zwischen den laizistischen Mächten (vor allem Militär, CHP und die Mehrheit der türkischen Bürokratie) einerseits und den islamistischen Bewegungen wie der politischen Partei RP von Erbakan und der derzeit regierenden AKP von Erdoğan andererseits veranlasste, ermöglichte, und rechtfertigte auch in den neunziger Jahren die permanente Einflussnahme des Militärs in Regierungsangelegenheiten. Das Paradox besteht darin, dass Staatskemalisten - vor allem das Militär - das Laizismusprinzip als untrennbaren Bestandteil einer funktionierenden Demokratie sehen und daher die Rolle des Militärs in der Politik als quasi-legitim betrachten, da dadurch eine Garantie gegen die Islamisierung des politischen Systems gewährleistet sei. Dabei begreifen sie den Laizismus als eine vom Staat ergriffene Maßnahme gegen die Einflussnahme der Islamisten, und übersehen dabei den viel wichtigeren Prozesscharakter der sozialen Säkularisierung in der türkischen Gesellschaft, die allerdings wiederum größtenteils als Verdienst des republikanischen Staatslaizismus begriffen werden soll. Der relevante Punkt in diesem Zusammenhang ist die oben erwähnte Legitimation der Macht des Militärs. Genauso wie der ethnische Separatismus im Südosten des Landes, der bis zur Mitte der 90er Jahre von den türkischen Entscheidungsträgern und Führungseliten als eine ernsthafte sicherheitspolitische Gefahr wahrgenommen wurde und daher die Rolle des Militärs - parallel zu seiner Funktion in der Sicherheitspolitik - in den sicherheitspolitischen Fragen quasi rechtfertigte und legitimierte, trägt die aus Sicht der hochrangigen Offiziere systemfeindliche Tendenz der islamistischen Parteien wie RP von Erbakan und AKP von Erdoğan zu den die Rolle des Militärs in diesem Kontext hinnehmenden Perzeptionen der staatslaizistisch orientierten zivilen Mächten (vor allem der linken Parteien) bei. Auch zahlreiche staatliche und nichtstaatliche Organisationen und Einrichtungen im Lande - vor allem die Universitäten - betrachten den Laizismus als die wichtigste Säule der türkischen Demokratie und positionieren sich gegen eine islamistische Relativierung des säkularen Charakters des republikanischen politischen Systems. Die Islamisten scheinen in der Tat trotz der negativen Erfahrungen der Vergangenheit die Tendenz zur Förderung der Islamisierung einiger Politikfelder, z.B. im Bereich der Bildungs- und Hochschulpolitik oder in der Geschlechterpolitik, nicht aufgegeben zu haben. Dennoch distanziert sich die eher muslimdemokratisch $^{8}$ und im Zusammenhang der türkischen Außenpolitik deutlich proeuropäisch orientierte AKP von Erdoğan von der auch im außenpolitischen Sinne is-

7 Ein Beispiel in diesem Bereich bildet ihre negative Haltung gegenüber den staatlichen Maßnahmen zur Förderung der Gleichstellung der Frauen in allen Gesellschaftsbereichen.

8 Dieser eher in Europa verwendete Begriff wird auch zunehmend in der türkischen Politikterminologie, vor allem in den Tageszeitungen und anderen Medien, verwendet. 
lamistisch orientierten fundamentalistischen RP von Erbakan. Die Spitzenpolitiker der AKP, vor allem die zur ehemaligen zweiten Generation der islamistischen MSPRP-Tradition gehörenden Führungseliten Erdoğan und Gül, wollen als eine konservative zentralrechte Richtung in der türkischen Parteienlandschaft begriffen werden. Dies erfordert natürlich eine gewisse Transformation der Bewegung. Es lässt sich die Frage nur in der Zukunft beantworten, ob es der AKP gelingt, sich von ihren eher fundamentalistisch orientierten Wurzeln (und Wählern) deutlicher zu distanzieren und sich zu einer muslimdemokratischen Partei zu transformieren, d.h. zu einer Partei, die Demokratie trotz ihrer muslimischen Wurzeln als Staatsform akzeptiert, und die in der Parteienlandschaft des politischen Systems in der Türkei einen festen Platz hat. Islamistische Nuancen - wie bei der Thematik der Imam-Berufsschulen - fallen in der Exekutivpraxis der AKP-Regierung auf. Sie implizieren ein gewisses Konfliktpotenzial mit dem bestehenden säkularen politischen System des Landes und dessen Verfassung. Die AKP wird in Zukunft umso mehr politisches Gewicht erhalten, je mehr sie sich in das politische System des Landes integriert. Eine mit dem System versöhnte muslim-demokratisch orientierte AKP könnte zur inneren Stabilität der Türkei viel beitragen. Dies würde die Position der Türkei in ihrer Rolle als demokratisches Beispiel für die anderen muslimischen Gesellschaften stärken.

Eine weitere außenpolitisch relevante ideologische Position stellt der Nationalismus als turkistische bzw. panturkistische Ideologie dar. Der Turkismus oder Panturkismus, der entstehungsgeschichtlich bis zum Desintegrationsprozess des Osmanischen Imperiums zurückverfolgt werden kann, gewann nach der Entstehung der postsowjetischen Turkrepubliken in der außenpolitischen Praxis an Bedeutung. Der (Pan)Turkismus als Gedankengut, der einerseits infolge der antikommunistischen bzw. anti-linken Tendenz seiner Anhänger - vor allem der MHP und ihrer pro-faschistischen bzw. rechtsextremistischen Grauen Wölfe - und aufgrund der realpolitischen Lage der turksprachigen Völker in der Sowjetunion während des Ost-WestKonflikts bis in die neunziger Jahre eher als eine reaktionäre und rechtsextremistische politische Position bezeichnet werden kann ${ }^{9}$ und andererseits das politische System nach dem Staatsstreich von 1980, insbesondere aber die Bildungspolitik, zum Teil dominierend beeinflussen konnte, wurde gleich nach der Desintegration der Sowjetunion zu einer endogenen Determinante der türkischen Regionalpolitik im Kaukasus und in Zentralasien. Zahlreiche (pan)turkistische Entscheidungsträger der konservativen Mitte-Rechts-Parteien (der DYP und der ANAP) übten Einfluss auf die öffentliche Perzeption in der Türkei bezüglich der Turkvölker aus. (Pan)Turkistische Bezüge wie die sprachlichen, kulturellen, historischen oder religiösen Gemeinsamkeiten zwischen den Türken und den postsowjetischen Turkvölkern wurden von führenden türkischen Politikern wie Özal oder Demirel sowie auch von anderen politischen Führungseliten - unter anderen auch den linken Politikern wie Ecevit - auch im Kontext der türkischen Beziehungen zu den postsowje-

9 Für die Einzelheiten vgl. Tanıl Bora, / Kemal Can, Devlet, Ocak, Dergah. 12 Eylül'den 1990'lara Ülkücü Hareket, Istanbul 1994. 
tischen Turkrepubliken ausgesprochen. Es ist möglich, in der türkischen Kulturpolitik im Kaukasus und in Zentralasien latente (pan)turkistische Tendenzen festzustellen, obwohl die türkischen Entscheidungsträger dies bisher stets kategorisch verneint haben. Auch realpolitisch nicht zu verwirklichende Tendenzen, engere Kooperationen mit den Turkrepubliken zu realisieren, erscheinen einen latent (pan)turkistischen Charakter zu implizieren. Es muss jedoch darauf hingewiesen werden, dass der Panturkismus infolge des osmanischen Expansionismus im Ersten Weltkrieg und dessen tragischen Folgen in der alltäglichen türkischen Politikterminologie mit seinem irredentistischen und expansionistischen Charakter im Vordergrund steht, wovon sich die republikanischen Entscheidungsträger bewusst distanzieren. Doch wie oben dargelegt, existieren in der ideologischen Kategorie der (Pan)Turkismen zahlreiche Tätigkeitsfelder. Im Bereich der Kultur bzw. der auswärtigen Kulturpolitik lässt sich der (Pan)Turkismus als politische Instrumentalisierung der kulturellen Gemeinsamkeiten und Ähnlichkeiten und deren gezielte Förderung definieren. Die türkische Unterstützung der Einführung des lateinischen Alphabets in den Turkrepubliken, um die bestehenden Gemeinsamkeiten zwischen dem Türkischen und den Turksprachen durch eine gezielte sprachliche Annäherung zu fördern, ist ein Beispiel dafür. Durch diese außenpolitische Instrumentalisierung des (Pan)Turkismus begründeten die türkischen Entscheidungsträger die intensiven Beziehungen zu den postsowjetischen Turkrepubliken, die im Rahmen der neuen Regionalpolitik einen besonderen Platz einnahmen.

\section{Rolle des innenpolitischen Erbes in der Außenpolitik}

Die zivilen türkischen Entscheidungsträger waren im Analysezeitraum auch mit den Schwierigkeiten des innenpolitischen Erbes des Staatsstreichs von 1980 konfrontiert. Durch die verfassungsmäßige Ermächtigung des Nationalen Sicherheitsrats (NSR) als ein quasi-exekutives Organ, ${ }^{10}$ in dem die militärischen Mitglieder gegenüber den Regierungsmitgliedern lange Zeit zahlenmäßig überlegen und vor allem gleichberechtigt in einem Gremium saßen, ermöglichte dem Militär ein institutionalisiertes Mitspracherecht. Das Militär konnte dadurch lange ein latentes Vetoregime im Sinne von Hale anwenden. ${ }^{11}$ Dieser Zustand wurde auch in den regelmäßigen Berichten der EU als eine Anomalie bezeichnet, weil die Macht der Exekutive von der Staatsbürokratie relativiert wurde. ${ }^{12}$ Es entstand ein Spannungsfeld zwischen den Gewählten (Regierung und Parlament) und den Ernannten (Militär und Staatsbürokratie).

10 George Harris, The Role of the Military in Turkey in the 1980s: Guardians or DecisionMakers? in: Metin Heper / Ahmet Evin, (Hg.), State, Democracy and the Military, New-York \& Berlin 1988, S. 177-200.

11 William Hale, Turkish Military and Politics, London 1994.

12 Siehe Regelmäßiger Bericht der EU-Kommission. Über die Fortschritte der Türkei auf dem Weg zum Beitritt, (1998; 1999; 2000; 2001; 2002; 2003) 
Diese Entwicklung hatte konkrete negative Folgen bezüglich der Rechtsstaatlichkeit und demokratischer Grundordnung. Dieses vom Militär dominierte Konzept spiegelt sich vor allem beim erweiterten Begriff der Sicherbeit in der politischen Praxis des Landes wider. Demnach wird die Außenpolitik, deren Kern nach diesem Konzept die Sicherheitspolitik bildet, überwiegend als ein überparteiisches und nationales Politikfeld betrachtet, ${ }^{13}$ in dem die dominante Rolle des NSR verfassungsrechtlich vorgesehen war. Einige außenpolitisch relevante Themen wie die türkische Zypernpolitik, Politiken bezüglich der Frage des kurdischen Separatismus und insbesondere Irak- und Armenienpolitik der Türkei gehörten lange zum Kompetenzbereich des militärisch dominierten NSR. Natürlich bedeutete diese Entpolitisierung der Außenpolitik und anderer Politikbereiche zweifelsohne eine offensichtliche Einschränkung der Machtkompetenz der Regierung. Bis zu den jüngsten Reformen zur Erfüllung der EU-Beitrittskriterien dauerte diese Funktion des NSR.

Seit Mitte der neunziger Jahre bis 2004 wurden zahlreiche Artikel der türkischen Verfassung von 1982 mitsamt ihrer Präambel revidiert und neu geschrieben. Nach den parallel zu diesen Verfassungsreformen durchgeführten demokratisierenden Reformpaketen wurde vor allem der NSR weitgehend von einem Organ im Entscheidungszentrum zu einem beratenden Gremium ohne politische Kompetenzen transformiert. Dadurch wurde die Legitimität der dualen Struktur des türkischen Entscheidungszentrums zumindest de jure beendet und somit wurden 2004 nach einem diesbezüglichen Bericht der EU-Kommission formell die EU-Kriterien von Kopenhagen erfüllt. Auch in der politischen Praxis wird seit der Neugestaltung des NSR eine Änderung des Verhältnisses zwischen der Regierung und dem Militär beobachtet.

Es lässt sich also feststellen, dass in diesem Prozess der Einfluss des Militärs parallel zu den demokratisierenden Reformen kontinuierlich abnimmt. Im außenpolitischen Zusammenhang ist diese Entwicklung insbesondere im Prozess der türkischen Integration in die EU von großer Bedeutung, zumal das entpolitisierte Politikfeld Außenpolitik von seinem militärisch dominierten Charakter befreit, die zivile Kontrolle in diesem Politikfeld gewährleistet und somit eines der problematischsten Bereiche im EU-Beitrittsprozess der Türkei weitgehend beseitigt wurde. Die Zyperngespräche im April 2004 zeigten deutlich, dass die Regierung - vor allem infolge der Neugestaltung des NSR - in der Lage ist, sogar bei der sicherheitspolitisch höchst sensiblen Thematik Zypern den politischen Willen auch gegen abweichende Perzeptionen der Armeeführung im NSR durchzusetzen und die Verantwortung der resultierenden politischen Entscheidungen alleine zu tragen. Daher darf dieser Schritt in der politischen Praxis nicht unterschätzt werden. Bisher scheint auch die Armeespitze ihre neue entpolitisierte Rolle akzeptiert zu haben.

13 Gencer Özcan, »Türkiye'de Siyasal Rejim ve Dıș Politika (1983-1993)«, in: Faruk Sönmezoğlu, Türk Dış Politikasının Analizi, Istanbul 1994, S. 293-315. 
Die Vorbereitung der Türkei auf den Beitritt zur EU im Rahmen der Beitrittspartnerschaft fördert nicht nur den Reformprozess zur Demokratisierung, sondern sie zwingt auch alle Führungseliten des Landes, darunter auch die militärischen Führungseliten, in der Praxis zu einem Wandel ihres politischen Verhaltens. Es bleibt abzuwarten, wie dieses neue Entscheidungszentrum, in dem das Militär über keine legitime oder legale politische Rolle oder Einflussmöglichkeit mehr verfügt, in der politischen Praxis funktionieren wird. Die Transformation im politischen System des Landes und vor allem in der Funktionsweise der Entscheidungsprozesse birgt natürlich ein gewisses Konfliktpotenzial zwischen den Zivilen und den Militärs, doch angesichts des bisher verwirklichten Demokratiestandes der Türkei und der zunehmenden Einflussnahme Europas in der türkischen Innenpolitik parallel zur zunehmenden Integration der Türkei in die EU wird die Möglichkeit eines Abbruchs der demokratischen Ordnung in der Türkei durch eine militärische Intervention ziemlich unwahrscheinlich.

\section{Beziebungen der Türkei zur Europäischen Union}

Die türkische Integration in die EU ist eines der aktuellen Themenbereiche der türkischen Außenpolitik. Der gesellschaftliche Konsens in der Türkei, die EU-Mitgliedschaft zu verwirklichen, spielt insbesondere seit dem Gipfel von Helsinki (1999), an dem die Türkei von der EU als Beitrittskandidat anerkannt wurde, in der türkischen Innenpolitik eine zunehmende Rolle. Der Prozess der Beitrittsvorbereitung fördert - als Instrument der EU wie bei anderen Beitrittskandidaten - den Demokratisierungsprozess in der Türkei.

Die Beziehungen der Türkei zu Europa einerseits und die EU-Kandidatur dieses Landes andererseits kann man als Kontinuität der türkischen Außenpolitik bezeichnen. Vor allem in der Außenpolitik der republikanischen Geschichte vom Ende des Zweiten Weltkriegs bis heute wird in der Tat eine unübersehbare Europäisierung der Türkei und kontinuierliche Integrationspolitik mit den westlichen bzw. europäischen Kooperationsformen festgestellt. Sicherlich ist die EWG/EG/EU-Mitgliedschaft und diesbezügliche Politik der Türkei der wichtigste Bestandteil der außenpolitischen Orientierung. Die EU-Orientierung der Türkei hat aus türkischer Perspektive zahlreiche Dimensionen. Die geographische Lage des Landes zwischen Europa, dem Kaukasus, dem nordöstlichen Mittelmeer, der Schwarzmeerregion und dem Nahen Osten sowie der identitätsbezogene kulturelle Dualismus zwischen islamischen Wurzeln und moderner europäischer Identität spielen nicht nur in Bezug auf die innenpolitischen Verhältnisse, sondern auch in den Beziehungen zwischen der Türkei und der EU eine relevante Rolle.

Dies hat historische Gründe: Interaktionen zwischen dem Osmanischen Reich und den anderen europäischen Mächten sowie die Französische Revolution und ihre politischen sowie kulturellen Folgen beeinflussten sowohl die Führungseliten und Intellektuellen der osmanischen Gesellschaft wie auch infolge der Entstehung der Nationalismen das Schicksal des Imperiums selbst. Gesellschaftliche Bereiche wie das Militärwesen (europäische Neugestaltung und Lehrprogramme), das politi- 
sche System (Relativierung des Absolutismus und Teilung der Macht mit dem Parlament), das Rechtssystem (Relativierung der islamischen Gesetzgebung und Einführung der europäischen Gesetze), das Schulsystem (Modernisierung der Schulen) etc. wurden ab dem 18. Jahrhundert von europäischen Entwicklungen und von Europa selbst immer stärker beeinflusst. ${ }^{14}$ Dies setzte in der osmanisch-türkischen Geschichte zugleich eine von Oben geleitete Modernisierung in Gang, die von den führenden Gesellschaftsschichten gefördert und von der Bevölkerung überwiegend verinnerlicht wurde.

Diese tradierte reformistische und europäisierende Haltung der türkischen Führungsschichten wurde von den Gründern der republikanischen Türkei ererbt. Die Analyse der republikanischen Geschichte der Türkei lässt zweifelsohne feststellen, dass die Republik Türkei auf die ideelle Grundlage der europäischen Aufklärung gegründet wurde. Die Überreste islamischer Referenz(quellen) in der Politik, im Rechtswesen - sowohl im Sinne der Gesetzgebung, als auch in der Rechtspraxis und im öffentlichen Leben (d.h. in der nichtreligiösen Sphäre) wurden durch die Republikgründung auf revolutionäre Weise abgeschafft und die Rolle der Religion(en) durch den Staatslaizismus auf das Privatleben des Individuums beschränkt. Durch die Gründung der Republik im Jahr 1923 erfolgte eine rasche Europäisierung.

Außenpolitisch ist die Türkei wie ihr Vorgänger ein Teil der europäischen Staatenwelt. Während des Zweiten Weltkriegs agierte die Türkei trotz der Neutralitätspolitik ihrer Entscheidungsträger als ein europäischer Staat. In diesem Sinne war die Beteiligung oder Nicht-Beteiligung der Türkei am Zweiten Weltkrieg eine europäische Frage, die für die Entwicklung des Kriegs in Europa von großer Bedeutung war. Nach dem Zweiten Weltkrieg war sie ebenfalls Teil der Nachkriegsordnung Europas: sie erhielt beispielsweise eine Wirtschaftshilfe im Rahmen der TrumanDoktrin (1947) und wurde von den USA und von ihren europäischen Verbündeten als Teil des (west)europäischen Staatensystems angesehen. ${ }^{15}$ Nach der Konkretisierung der sowjetischen Expansionspolitik gegenüber der Türkei zeigten auch die türkischen Entscheidungsträger ein deutliches Interesse, mit den USA und Westeuropa in der Sicherheits- und Militärpolitik eng zu kooperieren. Die türkische Rolle im Ost-West-Konflikt in Südosteuropa und in der nordöstlichen Mittelmeerregion trug erheblich zur westeuropäischen Sicherheit bei und verstärkte die Selbstperzeption der Türken als Europäer weiter, was den intensiven Kooperationen mit Europäern im Bereich der Sicherheitspolitik und zunehmend auch in wirtschaftlichen und politischen Bereichen entsprach.

Da diese sicherheitspolitische Notwendigkeit der türkischen Integration in Europa nicht mehr bestand, erhielt die Türkei von der EG/EU zu Beginn der 90er Jahre

14 Für die Einzelheiten siehe Bernard Lewis, The Emergence of Modern Turkey, London 1968.

15 Für die türkische Außenpolitik in diesem Zeitraum siehe Hüseyin Bağc1, Die türkische Außenpolitik wäbrend der Regierungszeit Menderes von 1950 bis 1960, Bonn 1988, S. $11 \mathrm{ff}$. 
keine konkrete Beitrittsperspektive, während den ehemalig sozialistischen Staaten Osteuropas von der EU in absehbarer Zeit ein sicherer Platz in der künftigen Union reserviert wurde. Aufgrund dieser Tatsache musste sich die Türkei nun auf Teilbereiche der Integration mit der EU konzentrieren, um von der europäischen Integration nicht vollständig ausgeschlossen zu werden. In diesem Zusammenhang erschien die Intensivierung der bestehenden Integration im Rahmen der EUZollunion auf der vertraglichen Grundlage des EWG-Türkei-Assoziationsabkommens von Ankara (1963) ein alternativer Weg zu sein, eine Art Sonderbeziehung zur EU zu etablieren mit der Hoffnung, durch den ökonomischen Spill-Over-Effekt künftig in weiteren Politikfeldern besondere Integrationsmöglichkeiten zu erhalten und Beziehungen zur Union weiter zu intensivieren. 1995 wurde nach langwierigen Verhandlungen der Vertrag der Zollunion unterzeichnet, und 1996 trat die Türkei der Europäischen Zollunion bei. Im Hinblick auf die Demonstration der türkischen EU-Orientierung war der Beitritt in die EU-Zollunion - ohne jedoch an Entscheidungsmechanismen der EU teilnehmen zu können, d.h. ohne zuvor Vollmitglied zu werden - von weitreichender politischer Relevanz, da die Türkei im Rahmen dieser besonderen Beziehung zur EU auf gewisse Souveränitätsrechte einseitig verzichtet hat. Dadurch übernahm die Türkei als erster Staat den EU-Außenzolltarif für dritte Staaten, ohne zuvor der EU beigetreten zu sein. ${ }^{16}$

Die türkischen Entscheidungsträger und Führungseliten definierten zu dieser Zeit die Aufnahme ihres Landes in den Kreis der offiziellen EU-Anwärter bzw. die offizielle Beitrittskandidatur als primäres außenpolitisches Ziel in der Europapolitik. Die Staats- und Regierungschefs der Union tendierten jedoch dazu, die Türkei nicht als Beitrittskandidat anzuerkennen und Zugeständnisse für die Entwicklung der Beziehungen in Richtung Vollmitgliedschaft zu machen, aber andererseits - wie durch die Zollunion mit der Türkei - das "prowestliche Land « mit politischen Schritten in einer Art Sonderbeziehung an Europa zu binden, da im Hinblick auf die europäische Sicherheitspolitik und auf seinen wachsenden konsumorientierten Binnenmarkt ein Land wie die Türkei relevant war. Zahlreiche Regierungen in der EU - z.B. die damalige Bundesregierung und der Bundeskanzler Kohl - nahmen die Türkei als ein Land wahr, das trotz seiner partiellen Zugehörigkeit zu Europa kein kultureller Teil Europas ist. Diese die Türkei aufgrund der Religion, Kultur und Geschichte ablehnende Position der Christdemokraten in der EU führte $1997 \mathrm{zu}$ einer Erklärung der Europäischen Volkspartei, dass die Türkei nicht als EU-Kandidat in Frage käme, da sie als islamisches Land über andere kulturelle Werte verfüge und daher nicht zur europäischen Zivilisation passe. ${ }^{17}$ Diese Huntington'sche Denkweise dominierte die damalige EU und mündete nach dem EU-Gipfel von Luxemburg im Jahr 1997 in die bisher tiefste Krise in der Geschichte der türkisch-europäischen

16 Șefik Alp Bahadir, »Die Zollunion der Türkei mit der Europäischen Union - ein Schritt auf dem Weg zur Vollmitgliedschaft? « in: Aus Politik und Zeitgeschichte, B 11-12/97, 7. März 1997, S. 33-40; S. 33ff.

17 Süddeutsche Zeitung vom 22. März 1997. 
Beziehungen. Die Krise nach Luxemburg zeigte, dass Perzeptionsunterschiede in den türkisch-europäischen Beziehungen ein ernst zu nehmendes Konfliktpotential bilden.

Derartige Perzeptionsunterschiede zwischen den türkischen Führungseliten und den europäischen Entscheidungsträgern existierten zwar, wie oben angesprochen, auch während des Ost-West-Konflikts, aber sie beeinflussten die sicherheitspolitisch definierte Lage der Türkei im westlichen Bündnis - also auch in Europa während des Ost-West-Konflikts nicht auf bestimmende Weise. Darüber hinaus war die Türkei aus Sicht der europäischen Entscheidungsträger zum einen angesichts ihrer ökonomischen und politischen Lage von einem Beitritt weit entfernt und zum anderen erforderten die außerordentlich wichtige Rolle des Landes im Ost-West-Konflikt sowie die Rahmenbedingungen der europäischen Konstellation auf dem geteilten Kontinent nicht unbedingt eine Manifestation und Verdeutlichung derartiger Perzeptionsunterschiede. Die europäischen Entscheidungsträger betrachteten die Türkei zwar aus realpolitischen Gründen immer als einen zuverlässigen Partner im Rahmen der blockgebundenen euro-atlantischen Sicherheitspolitik und unterstützten sie aus diesem Blickwinkel auch bei der Frage ihrer Europaorientierung, sie nahmen sie sogar im Zusammenhang der Erweiterung zusammen mit anderen euromediterranen Ländern wie Griechenland, Spanien oder Portugal wahr, die ähnliche ökonomische und soziopolitische Probleme hatten, aber die Europaintegration der Türkei konnte nicht wie bei anderen euromediterranen Ländern zum Beitritt führen.

Die Türkei fand sich insbesondere bezüglich ihrer innenpolitischen Lage auf die neue internationale Konstellation nach dem Ende des Ost-West-Konflikts ziemlich unvorbereitet: das Erbe der Militärregierung zwischen 1980-1982 und die als dessen Folge verabschiedete Verfassung von 1982 verhinderten bzw. verlangsamten den Demokratisierungsprozess in der Türkei und verursachten eine ernsthafte Instabilität in der türkischen Innenpolitik. Von Beginn der 80er Jahre an wurde diese Problematik zu einem der wesentlichen Themen in den türkisch-europäischen Beziehungen, das wegen seiner Relevanz zunehmend eine ausschlaggebende Rolle zu spielen begann.

Während es in den 90er Jahren den ehemals sozialistischen mittel- und osteuropäischen Staaten gelang, ihre politischen und wirtschaftlichen Transformationsprozesse erfolgreich abzuschließen und von der EU konkrete Beitrittsperspektiven angeboten zu bekommen, erwiesen sich die türkischen Fortschritte vorwiegend als ungenügend. Die fehlende konkrete Beitrittsperspektive der Türkei erwies sich in diesem Zusammenhang äußerst kontraproduktiv. Die Türkei gehörte zu dieser Zeit offensichtlich nicht zu den Prioritäten der EU-Erweiterung.

Die türkischen Entscheidungsträger und Führungseliten waren mit dem Problem konfrontiert, dass ihr Land in Europa zunehmend an die Peripherie gerückt wurde. Die Desintegration der Sowjetunion sowie die Befreiung - und Europaorientierung - ihrer Satellitenstaaten in Osteuropa ermöglichte der EU, neue Visionen für die politische Gestaltung des europäischen Kontinents zu entwickeln und zum Teil zu realisieren, wobei die geographische Entfernung der Türkei von Kerneuropa sich 
bezüglich der türkischen Interessen weitgehend als nachteilig erwies. Die neuen Dimensionen der europäischen Integration sahen neben der Vertiefung der wirtschaftlichen Integration auch den zunehmenden Ausbau der politischen, außen- und sicherheitspolitischen Komponente vor und verstärkten die Position der EU in der Weltpolitik. Von dieser neuen integrationspolitischen Gewichtung der EU dürfte sich die Türkei aus Sicht der türkischen Entscheidungsträger vor allem aufgrund ihrer intensiven ökonomischen, politischen und sicherheitspolitischen Beziehungen nicht isolieren. In diesem Sinne wurde der Wertverlust des Landes in Bezug auf die europäische Integration von den türkischen Entscheidungsträgern als eine äußerst negative außenpolitische Ausgangslage und Entwicklung bewertet. Die Entscheidung von Luxemburg (1997) wurde vom amtierenden Regierungschef Yilmaz als Diskriminierung bezeichnet.

Die aus türkischer Sicht diskriminierende Politik der EU gegenüber der Türkei wurde quer durch alle Gesellschaftskreise, in Hochschulen, von nichtstaatlichen Organisationen und von allen Proeuropäern scharf kritisiert. Die Enttäuschung der türkischen Eliten war immens. Auch in der EU löste die unerwartete Reaktion der Türkei neue Diskussionen über die EU-Türkei-Beziehungen aus und setzte einen politischen Denkprozess in Gang. Eine Normalisierung der Beziehungen gelang erst nach dem Beschluss der Staats- und Regierungschefs der Mitgliedsstaaten der Union auf dem EU-Gipfel von Helsinki. Die dort getroffene Entscheidung, die Türkei offiziell zum Beitrittskandidaten der EU zu erklären und ihre Kandidatur auf der Grundlage der dafür allgemein gültigen Prinzipien und Kriterien zu bewerten, bestätigte die klare Beitrittsperspektive der Türkei und nahm das Land in den Erweiterungsprozess der EU auf. Die Entscheidung in Helsinki bestätigte die Zugehörigkeit der Türkei zu Europa und verdeutlichte die strategischen Interessen der EU an einer Integration der Türkei. ${ }^{18}$ In Helsinki erklärten Premierminister Ecevit (DSP) und der Außenminister Cem (DSP), die notwendigen Reformen zur Erfüllung der EU-Beitrittskriterien von Kopenhagen energisch voranzutreiben, um die Anpassung der Türkei an die EU-Normen zu beschleunigen. Der Reformprozess von 1999 bis Mitte 2004 brachte praktisch eine neue Verfassung hervor, die entsprechend den EU-Normen die Grundordnung einer europäischen Rechtsstaatlichkeit erfüllt. Ausgehend von diesen Verfassungsreformen fand in der Türkei eine politische Systemtransformation statt, die die letzten undemokratischen Bestandteile der Ordnung von 1982 abschaffte. Die politischen Beitrittskriterien von Kopenhagen wurden bis dato de jure weitgehend erfüllt. Auch die EU deutete bereits 2002 auf die Erfüllung der wesentlichen Prioritäten der Beitrittspartnerschaft hin. Aufgrund dieser positiven Entwicklungen eröffnete die EU 2005 Beitrittsverhandlungen mit der Türkei - trotz ihres betonten ergebnisoffenen Charakters - mit dem einzigen Ziel: Vollmitgliedschaft.

18 Ian O. Lesser, »Turkey in a Changing Security Environment«, in: Journal of International Affairs, Herbst, 54/2000, S. 183-198; S. 188. 
Die klare Beitrittsperspektive der Türkei beschleunigte, wie oben angedeutet, den türkischen Demokratisierungsprozess und bestätigte nochmals die stabilisierende politische Rolle der EU in Europa. Nicht nur im Hinblick auf die innenpolitischen Verhältnisse der Türkei, sondern auch auf die Außenpolitik des Landes wurde zwischen 1999-2004 der Einfluss der EU deutlich. Die türkische Haltung nach der USamerikanischen Invasion im Irak, d.h. der Dritte Irakkrieg, die deutliche Entspannung und der initialisierte Dialog in den türkisch-griechischen Beziehungen und vor allem der neue außenpolitische Kurs der Türkei in der Zypernfrage sind die greifbarsten Ergebnisse der türkischen Außenpolitik, in denen die Beeinflussung der EU am konkretesten feststellbar sind. Die Zustimmung zum Plan der UN zur Wiedervereinigung Zyperns wäre für die türkischen Entscheidungsträger ohne die klare EU-Beitrittsperspektive der Türkei undenkbar gewesen. Diese Kursänderung der Türkei reichte zwar nicht für die Wiedervereinigung der Mittelmeerinsel vor dem Beitritt des griechischen Teils zur EU, aber sie entspannte den Konflikt und stärkte die Positionen der EU und der UN. Von daher ist die wachsende Rolle der EU in der Konfliktkonstellation im nordöstlichen Mittelmeer als positiv zu bewerten. Die Entspannung in dieser Region zwischen dem EU-Mitglied Griechenland, dem neuen Mitglied der Union Zypern (de facto nur griechischer Teil) und dem EU-Beitrittskandidaten Türkei demonstriert die Möglichkeiten der europäischen GASP in ihren Grenzregionen.

Die Türkeipolitik der EU birgt trotz der diesem Land angebotenen Beitrittsperspektive einige Konfliktpotenziale. Diese hängen mit europäischen Türkei-Perzeptionen zusammen. In Europa wird die Türkei aufgrund ihrer mehrheitlich muslimischen Bevölkerung nicht als ein natürlicher Europäer wahrgenommen. Zahlreiche europäische Politiker vertreten ihre Turkofobie im Kontext der europäischen Einigung ohne Bedenken. ${ }^{19}$ Einige politische Parteien und Führungseliten in Deutschland und Frankreich fordern energisch die Revidierung des Status der Türkei als Beitrittskandidat und beabsichtigen, den türkischen Beitritt zu verhindern oder möglichst zu verzögern, selbst wenn die Türkei politisch und wirtschaftlich für einen Beitritt bereit wäre. Die christdemokratisch-konservativ orientierten Kräfte in Europa wollen der Türkei anstatt einer Vollmitgliedschaft eine Art Sonderbeziehung mit der EU anbieten. Trotz der bereits begonnenen Beitrittsverhandlungen steht diese die Türkei aus der europäischen Integration ausschließende Idee noch auf der Tagesordnung und verfügt über Potenzial, die Agenda der EU bezüglich ihrer Türkeipolitik jederzeit zu ändern.

Die angebotene Sonderbeziehung wird jedoch von den türkischen Führungseliten als eine höflich formulierte Ablehnung des türkischen Beitritts zur EU wahrgenommen. Diese Position, die als Zollunion plus zusammengefasst werden kann, ist zwar derzeit nicht die offizielle Türkeipolitik der EU, sie muss allerdings im $\mathrm{Zu}$ sammenhang mit den möglichen Entwicklungstendenzen der türkischen Integrati-

19 Vgl. Thomas Meyer, Die Identität Europas. Der EU eine Seele?, Frankfurt am Main 2004, S. 148. 
on in die EU mit einkalkuliert werden. Fakt ist, dass die Führungseliten aller Parteien des Landes eine Sonderbeziehung zwischen der Türkei und der EU einheitlich kategorisch ablehnen. Diesbezüglich gibt es also landesweit einen weitgehenden Konsens. Es ist kurz- und mittelfristig nicht zu erwarten, dass die türkischen Entscheidungsträger ihre politische Haltung gegenüber Alternativen der EU-Vollmitgliedschaft ändern. Im Falle einer Revidierung der EU-Entscheidung, die Türkei als zukünftiges Mitglied zu betrachten, könnten daher ernsthafte Belastungen in den Türkei-EU-Beziehungen herbeiführen. Es wäre denkbar, dass diese Probleme auch die bilateralen Beziehungen der Türkei zu den einzelnen EU-Staaten beeinträchtigen könnten. Eine Revidierung des Endzieles der Beitrittsverhandlungen in der EU würde darüber hinaus die Glaubwürdigkeit der EU nicht nur aus der Perspektive der türkischen Perzeptionen, sondern auch in der Welt erschüttern. Ferner könnte die Ablehnung der Türkei auf der Grundlage des religiös-kulturellen Unterschiedes der Türkei das EU-Bild in der muslimischen Welt negativ beeinflussen und zusätzliche Polarisierungen zwischen Europa - auch dem Westen allgemein - und dem Islam hervorrufen. Eine solche Entwicklung könnte auch die Erfolgchance eines von der Türkei repräsentierten säkular-demokratischen politischen Systems und einer liberal-pluralistischen Islamauffassung - was auch als Euro-Islam bezeichnet wird ${ }^{20}-$ vermindern. Auch für die türkischen Einwanderer in Europa wäre dies ein polarisierendes Signal. Auf längere Sicht könnte ein eindeutiger Ausschluss der Türkei von der europäischen Integration das Selbstverständnis der türkischen Bevölkerung verändern.

\section{Regionalpolitik nach dem Ost-West-Konflikt}

Die neue weltpolitische und regionale Konstellation sowie die daraus entstandene relative Peripherisierung der Türkei aus europäischer Perspektive verursachten zu Beginn zwar einen gewissen Wertverlust der Türkei im Hinblick auf die europäische Sicherheitspolitik, aber andererseits eröffneten sie für die Türkei neue Möglichkeiten und Chancen für die Entwicklung einer aktiven Regionalpolitik. Zahlreiche internationale Führungspersönlichkeiten wiesen der Türkei in diesem Kontext eine aktive regionale Rolle zu. Tatsächlich entstanden in der nahen Umwelt der Türkei - auf dem Balkan, im Schwarzmeerraum, im östlichen Mittelmeer und im Nahen Osten, aber vor allem im Kaukasus und in Zentralasien - neue wirtschaftliche sowie außen- und kulturpolitische Kooperationsmöglichkeiten und Betätigungsfelder. Insbesondere die neuen unabhängigen postsowjetischen Staaten im Kaukasus und in Zentralasien sollen in diesem Zusammenhang als ein vordringliches Betätigungsfeld der neuen türkischen Regionalpolitik betrachtet werden.

Es soll an dieser Stelle zunächst nochmals auf die Relevanz der Gestaltung einer neuen Regionalpolitik der Türkei betont werden. Es lässt sich - wie oben bereits an-

$20 \mathrm{Zu}$ diesem Begriff siehe Thomas Meyer, Die Identität Europas. Der EU eine Seele?, Frankfurt am Main 2004, S. 152-154. 
gedeutet - vorab feststellen, dass diese neue Regionalpolitik vor allem eine Notwendigkeit im Sinne der Anpassung der Türkei an die neue regionale Konstellation war. Dies kann folgendermaßen begründet werden: Die Desintegration der Sowjetunion war Ausgangspunkt für diese außenpolitische Notwendigkeit. Die direkte Nachbarschaft der Türken und Russen ging durch die Desintegration der Sowjetunion nach Jahrhunderten zu Ende. In dem ehemals russisch-sowjetischen Territorium im Südkaukasus, mit dem die Türkei eine lange Grenze hat, erlangten drei ehemals sowjetische Unionsrepubliken ihre Souveränität und wurden am Ende des Desintegrationsprozesses zu unabhängigen Staaten. Auf dem Balkan nahm der russische Einfluss parallel zu dieser Entwicklung deutlich ab. Die Region Südosteuropa geriet unter die starke Anziehung der EU. Im Schwarzmeerraum erlangten die sowjetischen Unionsrepubliken Ukraine und Moldawien ebenfalls ihre Unabhängigkeit. In Zentralasien - einer Region, die von den benachbarten Regionen der Türkei geographisch getrennt ist, zu der jedoch historische und kulturelle Verbindungen vorhanden sind - entstanden ebenfalls neue postsowjetische Republiken. Der Irak wurde nach dem Ende des Zweiten Irakkriegs de facto geteilt und demzufolge entstand im Nordirak aufgrund der Entmachtung der irakischen Autorität - eine Region, mit der die Türkei eine gemeinsame Grenze hat - ein Machtvakuum. Diese Ausgangslage der türkischen Regionalpolitik könnte folgendermaßen zusammengefasst werden: einerseits gab es für die türkische Außenpolitik neue Betätigungsfelder mit neuen Möglichkeiten und Optionen, von denen die Türkei mittel- und langfristig profitieren könnte. Andererseits aber wurde die türkische Außenpolitik durch die regionale Instabilität direkt bedroht. Es war jedenfalls für die türkischen Entscheidungsträger ein Faktum, dass die Rahmenbedingungen der türkischen Außenpolitik bis zum Ende des Ost-West-Konflikts ihre Gültigkeit voll und ganz verloren hatten. Sie mussten daher entsprechend den realpolitischen Rahmenbedingungen und Konditionen der neuen regionalen und weltpolitischen Konstellation eine neue regionale Außenpolitik gestalten. Ein außenpolitischer Anpassungsprozess musste in Gang gesetzt werden. Dieser Prozess erforderte eine neue Lagebeurteilung der regionalen Umwelt, eine Definition der neuen Interessen entsprechend den neuen regionalen Rahmenbedingungen und letztendlich die Gestaltung einer neuen Regionalpolitik. Die Zentralasien- und Kaukasuspolitik der Türkei war ein signifikanter Bestandteil der neuen türkischen Regionalpolitik nach dem Ende des Ost-WestKonflikts. Am Beispiel der Analyse dieser Neuorientierung können die fundamentalen Entwicklungen sowie deren mögliche Folgen und Entwicklungstendenzen der neuen türkischen Regionalpolitik festgestellt werden.

Bei der Bewertung der türkischen Zentralasien- und Kaukasuspolitik sollen die Perzeptionen der türkischen Entscheidungsträger mit berücksichtigt werden, da diese insbesondere in der Phase der Lagebeurteilung und der Interessensdefinition von entscheidender Relevanz sind. In der Türkei wurde die Auflösung der Sowjetunion nicht nur aufgrund des gesunkenen Gefahrenpotenzials im Hinblick auf die türkische Sicherheitspolitik begrüßt, sondern auch infolge der Entstehung der türkischen Welt. Diese subjektive Perzeption der türkischen Eliten ist einerseits auf mangelndes Wissen der Mehrheit der türkischen Eliten über die turksprachigen 
Völker der ehemaligen Sowjetunion, andererseits auf bewusste Propagandaarbeit der (pan)turkistischen Intellektuellen zurückzuführen. Es ist zwar eine Tatsache, dass es nicht zu unterschätzende sprachliche Ähnlichkeiten zwischen den Turksprachen - auch mit dem Türkischen - gibt, doch ein Vergleich deutet nicht auf bloße Dialektunterschiede hin, sondern - abgesehen der türkischen und aserbaidschanischen Sprachen - eher auf Sprachunterschiede. Die Perzeption der türkischen Entscheidungsträger unterschied sich in diesem Punkt deutlich von der den politischen Führern der postsowjetischen Turkrepubliken. In der Türkei wurden die turksprachigen Völker dieser Republiken ethnisch als zentralasiatische Türken (Orta Asya Türkleri) wahrgenommen. Die fehlende Unterscheidung im Türkischen zwischen Türkische Sprache und Turksprache sowie Türke und Turk trugen zu diesem begrifflichen Fehlgebrauch bei, wobei man jedoch hier auch eine ideologisch begründete bewusste Ignoranz nicht völlig ausschließen darf. Die für die Türkei nach der Desintegration der Sowjetunion entstandene außenpolitische Neuorientierungsmöglichkeit wurde rasch zum Thema rhetorischer Machtprojektionskapazitäten. Das Entstehen einer Einflussregion von der Adria bis zur Chinesischen Mauer ${ }^{21}$ war der politische Ausdruck der türkischen Euphorie. Nahezu alle türkischen Entscheidungsträger waren mehr oder weniger davon überzeugt, dass die Türkei in Eurasien das Potential hätte, sich zu einem mächtigeren regionalen Akteur zu entwickeln. Sie tendierten darüber hinaus eher dazu, die Türkei als einen »älteren Bruder« der Turkrepubliken wahrzunehmen. Sie betonten in diesem Zusammenhang die transepochale Staatlichkeit der Türken als Argument für ihre Perzeption. Als weitere Stärken des Landes wären vor allem sein demokratisches politisches System, seine liberale und exportorientierte Marktwirtschaft sowie vor allem seine Integration im Westen. Die türkischen Entscheidungsträger sahen für die Türkei auch ein Potenzial, die politischen Werte Europas durch ihre engeren Beziehungen zu den kaukasischen und zentralasiatischen Regionen zu verbreiten. Ausgehend von dieser Perzeption übernahm die Türkei insbesondere in der Anfangsphase der Beziehungen eine Vermittlerrolle zwischen den Turkrepubliken und den westlichen Institutionen, in denen sie Mitglied ist. ${ }^{22}$ Die Türkei war der erste Staat, der die Unabhängigkeit der postsowjetischen Turkrepubliken anerkannt hat. Auch die ersten Auslandsvertretungen in diesen Republiken waren die türkischen Botschaften. Der Südkaukasus und Zentralasien gehören darüber hinaus zu den von türkischen Politikern am meisten besuchten Regionen. Die primären türkischen Interessen können wie folgt zusammengefasst werden:

21 Kut weist darauf hin, dass der Ausdruck Türkische Welt von Adria bis China zum ersten Mal in der Weltwirtschaftsforum am 1. Februar 1992 vom US-amerikanischen Staatsmann Kissinger verwendet wurde. Auch das Buch von Graham Fuller und Ian O. Lesser hat den Untertitel From the Balkans to Western China. Siehe Gün Kut, »Yeni Türk Cumhuruyetleri ve Uluslararası Ortam«, in: T.C. Kültü̈r Bakanlı̨̆̌ (Hg.): Bağımsızlığın İlk Yılları, Ankara 1994, S. 9-24; S. 13.

22 Bess Brown / Elisabeth Fuller, Die Türkei und die muslimischen Republiken der ehemaligen Sowjetunion, Sankt Augustin 1994, S. $19 f$. 
1. Die Staatlichkeit und Unabhängigkeit der Turkrepubliken und der anderen postsowjetischen Staaten der Region sollen etabliert und gefestigt werden.

2. Eine politische Plattform zur Kooperation zwischen der Türkei und den Turkrepubliken soll initialisiert werden. Die Beziehungen zu den Turkrepubliken sollten möglichst institutionalisiert werden, um eine gewisse Kontinuität hervorzubringen.

3. Die kulturellen Ähnlichkeiten - insbesondere die sprachlichen, aber auch die historischen, religiösen und gesellschaftlichen - sollen im Rahmen einer türkischen Kulturpolitik gezielt gefördert werden und die wichtigste Basis der außenpolitischen Aktivitäten der Türkei bilden.

4. Die wirtschaftlichen Kooperationen mit den postsowjetischen Republiken sollen unterstützt und gefördert werden. Hierbei soll die Türkei zu einem Knotenpunkt für die aus der kaspischen Region stammenden fossilen Brennstoffe werden, die durch Pipelines zu türkischen Mittelmeerhäfen transportierten werden sollen.

5. Zur Lösung der Konflikte und zur Behebung der Konfliktpotentiale zwischen den postsowjetischen Nachbarstaaten soll durch bilaterale und multilaterale Initiativen - vor allem ohne militärische Machtprojektion - beigetragen werden.

6. Das wichtigste Interesse der Türkei in der Kaukasus- und Turkrepublikenpolitik ist - entsprechend dem generellen regionalpolitischen Interesse des Landes - eine außenpolitische Anpassung an die Rahmenbedingungen der neuen regionalen Konstellation nach dem Ost-West-Konflikt. Diese Interessenslage bestimmte die bisherige Zentralasien- und Kaukasuspolitik der Türkei.

Die ersten Kontakte zu den Turkrepubliken wurden kurz vor der Auflösung der Sowjetunion hergestellt. Mehrere bilaterale und multilaterale Verträge zwischen der Türkei und den Turkrepubliken wurden nach der Erlangung der Unabhängigkeit dieser Staaten paraphiert. ${ }^{23}$ Die multilateralen Formen der Beziehungen zu den postsowjetischen Turkrepubliken erwiesen sich eher als nicht effektiv und konnten die politischen und wirtschaftlichen Kooperationsmöglichkeiten nicht hinreichend konkretisieren. Die Kooperationsformen wie die ECO oder die Turkstaatengipfel waren hinter den Erwartungen der türkischen Entscheidungsträger zurückgeblieben. Einerseits fehlten der Türkei die wirtschaftlichen Kapazitäten und insbesondere die erforderlichen politischen und sicherheitspolitischen Strukturen. In diesem Zusammenhang wäre nochmals auf die machtpolitische Lage der Russischen Föderation als Erbin der sowjetischen Supermacht hinzuweisen. Andererseits sind zwischen den Entscheidungsträgern und Führungseliten der Türkei und den turksprachigen Führungen einige tiefgreifende Interessensunterschiede festzustellen. Die Entscheidungsträger der Turkrepubliken zeigten keinerlei Interesse an einer supranational orientierten und ethnisch oder religiös fundierten - und eventuell panturkistischen - Kooperationsform der Turkstaaten, die von den türkischen Entschei-

23 Siehe hierzu T.C. Milli Eğitim Bakanlığı (Hg.), Türkiye ile Türk Cumburiyetleri ve Türk Topluluklari Arasinda Yapilan Antlasmalar, Iliskiler ve Faliyetler, Ankara 1993. 
dungsträgern auf den Gipfeltreffen der turksprachigen Staaten vorgeschlagen worden war. ${ }^{24}$ Das nicht konkretisierte und eher als eine Option wahrgenommene Ziel türkischer Entscheidungsträger, mit den Turkrepubliken zusammen eine türkische Liga der wirtschaftlichen, politischen und kulturellen Zusammenarbeit zu initialisieren, muss so aus türkischer Perzeption als ein Versuch gesehen werden, für die EU und die USA wieder an Bedeutung zu gewinnen. Nicht nur im Hinblick auf die fehlenden finanziellen Kapazitäten, sondern auch auf machtpolitische Gleichgewichte in der Region mussten Träume über eine supranational geprägte Kooperationsform zwischen der Türkei und den Turkrepubliken scheitern. Vor allem wäre ein derartiges Vorgehen nicht unbedingt mit den Interessen Russlands im Einklang und würde die ohnehin dominante russische Politik des nahen Auslands provozieren.

Im Rahmen der initialisierten Kulturpolitik als einen wichtigen Bestandteil der türkischen Regionalpolitik entwickelten sich intensive kulturelle Beziehungen zwischen der Türkei und den turksprachigen Republiken. Insbesondere die Sprach-, Bildungs- und Hochschulpolitik waren die Aktivitätsbereiche der türkischen Kulturpolitik, in denen eine kulturelle Annäherung zu diesen Republiken stattfinden konnte. ${ }^{25}$ In Aserbaidschan beispielsweise konnte das lateinische Alphabet aufgrund der aktiven kulturpolitischen Initiative der Türkei das von Russland durchgesetzte und bisher verwendete kyrillische Alphabet erfolgreich ablösen, was zweifelsohne zur sprachlichen Verständigung auf höherer Ebene - auch in Literatur und Wissenschaft - zwischen der Türkei und Aserbaidschan zunehmend beitragen wird. In anderen postsowjetischen Turkrepubliken wurde das lateinische Alphabet nur teilweise eingeführt. Auch der Einfluss der türkischen Medien - vor allem der türkischen Fernsehkanäle und Zeitungen - könnten in diesem Zusammenhang eine zusätzliche Annäherung fördern. Die türkischen Gymnasien und Hochschulen in den kaukasischen und zentralasiatischen Regionen sind ebenfalls als wichtige Komponenten der türkischen Kulturpolitik zu betrachten, die mittel- und längerfristig zu einem bestimmenden Faktor der kulturellen Beziehungen zwischen der Türkei und den postsowjetischen Staaten dieser Regionen werden können. Diese Schulen und Universitäten sind elitär orientiert und bilden Kinder und Jugendliche der höheren Bildungs- und Führungsschichten dieser Länder aus. Durch staatliche Stipendien ermöglichte die Türkei zahlreichen Studenten aus den Turkrepubliken überdies wissenschaftliche Studien an den türkischen Universitäten. Ähnliche Studienmöglichkeiten an den türkischen Militärakademien wurden ebenfalls angeboten. Der

24 Hierzu siehe Nursultan Nazarbayev, Yüzyılların Kavșağında, Ankara 1997, S. $200 f$. Siehe auch Ahmet Kuru, »Uluslararasi Ortam ve Bölgesel Entegrasyon Teorileri Isiginda Türk Birligi Meselesi«, in: Mim Kemal Öke, (Hg.), Gecis Sürecinde Orta Asya Türk Cumburuyetleri, Istanbul 1999, S. 152-210; S. 185.

25 Für die Einzelheiten siehe Çaman, Türkische Außenpolitik nach dem Ende des OstWest-Konflikts: Außenpolitische Kontinuität und Neworientierungen zwischen der EUIntegration und neuer Regionalpolitik, S. 292-301. Siehe auch Zakir B. Avşar, »Türk Cumhuriyetleri Arasında İletișim«, in: Avrasya Etüdleri, 3/1 (1996), S. 101-113. 
Türkei gelang es dabei, die Besonderheiten ihres laizistischen politischen Systems und ihrer Lebensweise - eine pluralistisch orientierte parlamentarische Demokratie und eine pluralistische sowie säkulare Gesellschaftsstruktur - den zukünftigen Führungsschichten der Turkrepubliken nahe zu bringen. Andererseits mussten die türkischen Entscheidungsträger registrieren, dass der kulturelle Einfluss Russlands in diesen Jahrhunderte lang russisch dominierten Regionen fest etabliert ist und damit das Kulturleben sowie die politische und gesellschaftliche Praxis dominiert. In diesem Zusammenhang muss die übergeordnete Rolle der russischen Kultur und Sprache sowohl in Zentralasien als auch im Südkaukasus insbesondere unter den Führungsschichten betont werden. Diese Tatsache mussten die türkischen Entscheidungsträger einsehen und hinnehmen.

Ein weiteres Kooperationsfeld war der Bereich der wirtschaftlichen Beziehungen. Die Regionen Kaukasus und Zentralasien wurden von Anfang an sowohl von den türkischen Führungseliten als auch von den privaten Investoren aus der Türkei als ein potenziell bedeutender Markt für die seit den 80er Jahren exportorientierte türkische Volkswirtschaft wahrgenommen. Darüber hinaus verfügten die postsowjetischen Staaten - vor allem Aserbaidschan, Kasachstan und Turkmenistan - über strategisch wichtige Rohstoffe wie Erdöl und Erdgas. Für die türkischen Entscheidungsträger war es relevant, die wirtschaftliche Entwicklungspolitik und die Reformen zur Systemtransformation in diesen Staaten zu unterstützen, um zu ihrer Souveränität und Unabhängigkeit beitragen zu können. Zu diesem Zweck stellte die Türkei Kredite und humanitäre Hilfe für die Turkrepubliken zur Verfügung und leistete technische Hilfe bei der Ausbildung von Führungspersonen der Wirtschaft. Zur Modernisierung der Infrastruktur der Turkrepubliken wurden von der Türkischen Telekom und auch aus dem Privatsektor insbesondere im Bereich der Telekommunikation technische Unterstützungen angeboten. Die türkischen Entscheidungsträger waren sich von Anfang an bewusst, dass die wirtschaftlichen Ressourcen der Türkei nur eine begrenzte Unterstützung im wirtschaftlichen Bereich zulassen würden. In den Turkrepubliken bilden auf der anderen Seite die strukturellen Wirtschaftsprobleme - z.B. ihre Abhängigkeit von der Russischen Föderation als Folge des sowjetischen Erbes, veraltete Technologie, fehlende Kapitalakkumulation und Know-how-Defizite - eine konkrete Barriere für die Intensivierung der Wirtschaftsbeziehungen. Nichtsdestotrotz sind die Investitionen des türkischen Privatsektors in Zentralasien und im Südkaukasus nicht zu unterschätzen.

Ein strategisches Interesse der Türkei im Rahmen ihrer Zentralasien- und Kaukasuspolitik war die Durchsetzung der von der Türkei vorgezogenen Transportwege zur Vermarktung von Erdgas und Erdöl aus Zentralasien und dem Kaukasus. Dieser Bereich ist als ein Querschnittsbereich zwischen den strategischen und wirtschaftlichen Interessen des Landes zu betrachten. Die türkischen Entscheidungsträger erwarteten vor allem vom Pipelineprojekt Baku-Ceyhan (über Georgien und die Türkei), das von der internationalen Gemeinschaft - vor allem von den USA und der EU - unterstützt wird und aserbaidschanisches Erdöl in den Weltmarkt, vor allem aber nach Europa transportieren wird, sowohl wirtschaftliche als auch politi- 
sche und strategische Vorteile. Insbesondere im Kontext der zukünftigen Entwicklungstendenzen der Türkei-EU-Beziehungen zeigt sich die strategische Dimension dieses Projektes, das größtenteils vollendet wurde. Die türkische Pipelinepolitik als ein relevanter Bestandteil der neuen Regionalpolitik erwies sich in diesem Zusammenhang als erfolgreich. Angesichts dieser Einschätzungen, Bewertungen und Fakten in diesem Bereich der Beziehungen kann eine vielversprechende Möglichkeit der Intensivierung der wirtschaftlichen Beziehungen der Türkei insbesondere zu Aserbaidschan und Georgien, ferner längerfristig auch zu Armenien, prognostiziert werden. Parallel dazu ist die Wahrscheinlichkeit hoch, dass der Einfluss der EU sich im Südkaukasus - vor allem im Falle eines türkischen EU-Beitritts - sowohl im wirtschaftlichen als auch im politischen Sinne intensivieren wird. Dies könnte längerfristig konflikteindämmende und stabilisierende Projektionen durch EU-Initiativen unter aktiver Teilnahme der Türkei ermöglichen.

Gerade hierin liegt ein weiteres türkisches Interesse. Sowohl Zentralasien als auch der Kaukasus sind konfliktbeladene Regionen. Die russische und sowjetische Fremdherrschaft in diesen Regionen hatte zur Folge, dass sich zahlreiche Konfliktpotentiale, die während der Sowjetzeit nur latent bestanden, nach der Desintegration der Sowjetunion als Konfliktherde manifestierten und durch ihre Eskalation die Stabilität der Region untergraben. Tschetschenien (Russische Föderation), Adscharien (Georgien) und Berg-Karabakh (Aserbaidschan-Armenien) sind die wichtigsten, aber nicht die einzigen Konflikte im Transkaukasus. Zwei dieser gefährlichen Konfliktherde befinden sich in unmittelbarer Nähe der Türkei und stellen ein akutes Eskalations- und Instabilitätspotenzial dar. Vor allem der Konflikt zwischen den Armeniern und Aserbaidschanern um das aserbaidschanische Gebiet Berg-Karabakh betrifft die türkische Regionalpolitik in vielerlei Hinsicht. Das schlechte Verhältnis zwischen der Türkei und Armenien ist auf die tragischen Ereignisse im Ersten Weltkrieg und auf die daraus entstandenen unterschiedlichen Perzeptionen dieser Tragödie zurückzuführen. Dies ist gewiss ein dominanter Faktor eines gefährlichen Ultranationalismus und türkenfeindlicher Tendenzen in der heutigen postsowjetischen Republik Armenien. Eine weitere Problematik, die das türkischarmenische Verhältnis im Südkaukasus erschwert, ist die Aggressionspolitik Armeniens gegenüber seinem Nachbar Aserbaidschan. An einer friedlichen Lösung dieses Konflikts ist die Türkei vor allem auch deshalb interessiert, damit sich endlich stabilere Verhältnisse im Südkaukasus etablieren. Die Friedensinitiativen der Türkei brachten allerdings in der Vergangenheit keine konkreten Erfolge. Eine aktive Initiative seitens der EU, die eine baldige Lösung des Konflikts herbeiführen könnte, erscheint im Moment vor allem kurzfristig nicht realistisch zu sein. Es ist jedoch durchaus möglich, dass die zunehmende Einflussnahme der EU sich zu einem stabilisierenden Faktor im Südkaukasus entwickeln könnte. Auch in diesem Punkt stimmen die Interessen der Türkei und der EU überein.

Die türkische Regionalpolitik hängt selbstverständlich mit dem außenpolitischen Verhalten anderer regionaler und internationaler Akteure zusammen. Die Interaktionen dieser Akteure einschließlich der Türkei sind weitere exogene Bestimmungsfaktoren der türkischen Zentralasien- und Kaukasuspolitik. Die Entwicklungstendenzen 
der Beziehungen der Türkei zu den postsowjetischen Turkrepubliken werden von den Folgen der Interaktionen dieser regionalen Akteure der Konstellation beeinflusst. Der Iran im Kaukasus sowie Pakistan und Indien in Zentralasien zeigen sich als regionale Akteure mit geringen Einflussmöglichkeiten und Kapazitäten. China verfügt über enormes ökonomisches Potential und könnte sich in diesem Sinne insbesondere hinsichtlich seiner Außenhandelspolitik zu einem einflussreicheren Akteur in Zentralasien entwickeln. Diese regionalen Akteure haben eine überwiegend multiethnische Bevölkerungsstruktur und sind so der Gefahr interethnischer Konflikte ausgesetzt. Die repressive Nationalitätenpolitik Chinas in Ostturkistan (in Westchina) gegenüber dem Turkvolk Uiguren und der uigurische Nationalismus sind die Determinanten des ethnischen Konfliktpotenzials im chinesischen Zentralasien. ${ }^{26}$ Die chinesischen Entscheidungsträger müssen dieses latente Konfliktpotential bei den chinesisch-zentralasiatischen Beziehungen stets berücksichtigen, $\mathrm{da}$ in Zentralasien eine nicht $\mathrm{zu}$ unterschätzende uigurische Minderheit lebt. Auch für den Iran bildet die Aseri-Minderheit an der iranisch-aserbaidschanischen Grenze ein ethnisches Konfliktpotential mit denkbaren Eskalationstendenzen, die sich parallel zur wirtschaftlichen Entwicklung und internationaler Integration Aserbaidschans zu einem wichtigen innenpolitischen Problem des Iran entwickeln könnte. Sowohl im Falle Chinas wie auch des Iran könnte die problematische Struktur der fehlenden pluralistischen Demokratie und Rechtsstaatlichkeit Nährboden für ethnische Konfliktpotentiale sein.

Nach dem Ende des Ost-West-Konflikts benahm sich die Russische Föderation als ein führender und äußerst aktiver Akteur in Zentralasien und im Kaukasus. Es kann davon ausgegangen werden, dass die Russische Föderation bisher der wichtigste exogene Einflussfaktor auf die türkische Regionalpolitik in Zentralasien und im Kaukasus war. Es könnte aus vielerlei Hinsicht von einer türkisch-russischen Rivalität in Zentralasien und im Kaukasus die Rede sein, deren Ursachen in den kontroversen Perzeptionen und daraus resultierenden unterschiedlichen Interessen der beiden regionalen Staaten zu suchen sind. Die russischen Entscheidungsträger und Führungseliten nehmen die südlichen postsowjetischen Staaten im Kaukasus und in Zentralasien im Rahmen des außenpolitischen Konzepts des naben Auslands wahr und betrachten diese Regionen als eine Art abhängige Einflusszone. ${ }^{27}$ Diese nostalgische russische Sichtweise ist bis zur Kolonialpolitik des Russischen Reichs und zu der sowjetischen Fremdherrschaft in diesen Regionen zurückzuverfolgen. ${ }^{28}$ Die rus-

$26 \mathrm{Zu}$ diesem Thema siehe Gudrun Wacker, »Xinjiang und die VR China. Zentrifugale und zentripetale Tendenzen in Chinas Nordwestregion « in: BIOST, 3/1995.

27 Graham E. Fuller, »Russia and Central Asia: Federation or Fault Line?« in: Michael Mandelbaum, (Hg.), Central Asia and the World. Kasakhstan, Usbekistan, Tajikistan, Kyrgystan and Turkmenistan, New York 1994, S. 94-129. Siehe auch Uwe Halbach, "Zwischen sheißem Krieg< und reingefrorenen Konflikten<. Russlands Politik im Kaukasus «, in: Osteuropa, 4/5, 51. Jahrgang, 2001, S. 481-494. Für die russische Außenpolitik in Zentralasien siehe Birgit Brauer / Beate Eschment, »Russlands Politik in Zentralasien«, in: Osteuropa, 4/5, 51. Jahrgang 2001, S. 495-508.

28 Schilling, Walter: »Rückkehr des Imperialismus im Kaukasus?«, in: Internationale Politik, 50/11 (1995), S. 45-50. 
sischen Führungseliten versuchen seit dem Zusammenbruch der Sowjetunion, die im völkerrechtlichen Sinne zu Ende gegangene russische Herrschaft in diesen Regionen zumindest durch eine besonders aktive Regionalpolitik fortzuführen. Die Etablierung der Unabhängigkeit der ehemals sowjetischen Republiken im russischen naben Ausland entspricht in diesem Zusammenhang nicht unbedingt den strategischen Interessen der Russischen Föderation. Dies ist dem oben erwähnten strategischen Interesse der Türkei diametral entgegengesetzt. Die russischen Führungseliten betrachten daher jede politische oder wirtschaftliche Kooperation der postsowjetischen Staaten südlich ihres Territoriums als eine negative Tendenz, die die russische Vorherrschaft und Dominanz in der Region relativieren würde. Es gelang den russischen Entscheidungsträgern bisher weitgehend, die Bemühungen der türkischen Entscheidungsträger zu neutralisieren, eine Plattform der Kooperation mit den Turkrepubliken in die Tat umzusetzen. Diese mussten die von der Russischen Föderation durchgesetzte Lage aus realpolitischen Gründen hinnehmen. Auf der anderen Seite aber gelang es der Türkei ihre Pipelinepolitik in Zusammenarbeit mit den USA, trotz der energischen Opposition der Russischen Föderation durchzusetzen.

Für die türkischen Entscheidungsträger erscheint es insgesamt realistischer sein, mit dem Westen, vor allem aber mit der EU neue Kooperationsfelder zu suchen, um ihre Interessen besser wahrnehmen zu können. Insbesondere ihre Perzeptionen bezüglich der Etablierung der Unabhängigkeit der südkaukasischen Staaten - aber auch der zentralasiatischen Staaten - stimmen mit denen der EU sowie der USA weitgehend überein. Auch im Sinne der Stabilisierung des Südkaukasus können deutliche Übereinstimmungen mit den Perzeptionen der EU festgestellt werden, da die EU neuerdings die südkaukasischen Staaten in ihre ausgeweitete Nachbarschaftspolitik aufnahm. Auch Georgien und Aserbaidschan signalisieren ihr Interesse an einer Beteiligung an der EU-Integration. Die Entscheidungsträger dieser Länder wissen, dass der Beitritt der Türkei zur EU sie zu direkten Nachbarn der EU machen würde, was für sie eine positive Entwicklung im Hinblick auf ihre wirtschaftliche und politische Stabilität wäre und zudem ihre Unabhängigkeit von der russischen Dominanz stärken würde. Die Führungen der postsowjetischen Staaten im Südkaukasus werden diese Möglichkeit bei ihren Beziehungen zur Türkei in naher und mittlerer Zukunft immer vordringlicherer berücksichtigen. Für die EU wird möglicherweise vor allem der Reichtum Aserbaidschans an fossilen Brennstoffen sowie deren Transport über Georgien und die Türkei nach Europa eine zunehmende zentrale Rolle in ihrer Türkei- und Kaukasuspolitik spielen. Zentralasien hingegen befindet sich verglichen mit dem Südkaukasus unter viel stärkerem russischem Einfluss. Die geographische Entfernung Zentralasiens von der Türkei und von Europa bildet eine große Barriere für eine Intensivierung der Beziehungen der EU zu dieser Region. Für die Türkei ist es abgesehen von ihrer erfolgreichen Kulturpolitik und ihren wirtschaftlichen Aktivitäten in naher und mittlerer Zukunft nicht allzu wahrscheinlich, in Zentralasien und insbesondere auf dessen Märkten ein Kooperationsplattform mit den Turkrepubliken zu realisieren. Ihre geographische Entfernung sowie die geringfügigen und konkurrenzunfähigen wirtschaftlichen Kapazitäten sind offensichtliche Nachteile der Türkei in ihrer Zentralasienpolitik. 


\section{Schluss}

Es wurden nach dem Ende des Ost-West-Konflikts folgende, für die GesamtauBenpolitik relevante Folgen der neuen Konstellation beobachtet, die die Neugestaltung der türkischen Außenpolitik auf bestimmende Weise mit beeinflussten. Die Sichtweise und dementsprechend die Lagebeurteilung der außenpolitischen Entscheidungsträger und Führungseliten der Türkei mussten sich aufgrund den neuen exogenen Determinanten nach dem Ende des Ost-West-Konflikts ändern. Infolge neuer Machtlagen in der regionalen und weltpolitischen Konstellation, insbesondere aufgrund der Desintegration der Sowjetunion und des Warschauer Paktes, entstanden für die Türkei neue, vorher nicht oder nur latent und vor allem realpolitisch unbedeutend wahrgenommene Interessensfelder mit nun bedeutend gewordenen politischen, ökonomischen, sicherheitspolitischen und kulturellen Dimensionen. In diesem Sinne wurden nach dem Paradigmenwechsel in der Weltpolitik neue Orientierungen in der türkischen Außenpolitik beobachtet. Eine aktive Balkanpolitik, die neue Kooperationspolitik am Schwarzmeer (eine von der Türkei initialisierte Kooperation mit den Schwarzmeer-Anrainerstaaten), eine neue Nahostpolitik (IrakKriege und deren Folgen im Hinblick auf die türkische Außen- und Sicherheitspolitik) und vor allem die Zentralasien- und Kaukasuspolitik oder anders ausgedrückt, die Turkrepublikenpolitik, gehören zu den außenpolitischen Neuorientierungen, die auch ein neues Konzept der Regionalpolitik ermöglichten. Die neue Konstellation hinsichtlich der sich geänderten Machtlage zwischen den an der regionalen Konstellation beteiligten - teilweise neu entstandenen - Akteuren brachte eine neue Machthierarchie hervor und die türkischen Entscheidungsträger mussten dies bei der Gestaltung der regional gerichteten außenpolitischen Neuorientierungen des Landes mitberücksichtigen. Nach der Desintegration der Sowjetunion fand nicht nur ein Machtkollaps, sondern in der Folge vor allem auch eine Machtverlagerung statt. Hierbei stellte sich die Russische Föderation als regionaler Akteur in den Regionen, in denen die Türkei in 90er Jahren außenpolitisch aktiv war - vor allem im Kaukasus und in Zentralasien - im Hinblick auf ihr Machtpotenzial als Erbin der Sowjetunion, d.h. als dominierender Machtfaktor, dar. Im normativen Bereich, was Ethik, Recht, Ideologie und ihre Auslegung betrifft, waren die Einflüsse der neuen Konstellation zunehmend spürbar. Das Ende des Ost-West-Konfliktes eröffnete für die Türkei einen Raum für eine ideologisch beeinflusste regionale Außenpolitik, die während des bipolaren Systems nicht vorstellbar war. In der bipolaren Weltpolitik spielte die Türkei im westlichen Bündnis eine entscheidende sicherheitspolitische Rolle, so daß eine regional ausgerichtete Außenpolitik des Landes lediglich bescheiden möglich war. Während die ideologischen Grundpositionen in den außenpolitischen (und auch in den innenpolitischen) Entscheidungen zunehmend als Einflussfaktor wirkten und im außenpolitischen Zusammenhang an Relevanz gewinnen konnten, brachten die gegensätzlichen ideologischen Positionen zwischen den Regierungen und staatlichen Institutionen (vor allem dem Militär) ein Spannungsfeld hervor, das im Hinblick auf den Prozess der politischen Entscheidungsfindung und vor allem auf das politische System des Landes von großer Bedeutung ist. Eine wei- 
tere Beobachtung im Zusammenhang der normativen Bindungen stellt der zunehmende Einfluss der Europäischen Union im Demokratisierungsprozess der Türkei gemäß den EU-Beitrittskriterien von Kopenhagen dar. Die kontinuierliche außenpolitische Europaorientierung mit dem Ziel der Vollmitgliedschaft in der EU brachte vor allem seit der zweiten Hälfte der 90er Jahre die zunehmende Übernahme der politischen Normen der EU hervor. Die gegenseitige Beeinflussung zwischen der Außen- und Innenpolitik war im Analysezeitraum charakteristisch in den TürkeiEuropa-Beziehungen. In diesem Prozess wurde die türkische Verfassung von 1982 sowie andere Gesetze so reformiert, daß das politische System des Landes sich in die Richtung von Demokratisierung und EU-Anpassung bewegte, sich damit liberalisierte und europäisierte.

Die Rahmenbedingungen der türkischen Außenpolitik änderten sich entsprechend den Konstellationsänderungen in der Weltpolitik in jeder Hinsicht, so dass die türkischen Entscheidungsträger und Führungseliten mit der ernsthaften Frage konfrontiert waren, die Außenpolitik der Türkei rasch an die neuen Rahmenbedingungen anzupassen und sie dementsprechend neu zu gestalten. Zu diesem Zweck versuchten sie vor allem, ein neues Konzept der Regionalpolitik parallel zu den aktuellen Rahmenbedingungen nach dem Ost-West-Konflikt zu entwickeln, um eine außenpolitische Anpassung an die geänderte Situation zu schaffen. Dies wurde von manchen Autoren auch als eine aktive Außenpolitik bezeichnet. In diesem Zusammenhang ist die neue türkische Regionalpolitik als eine Art präventiver Außenpolitik zu verstehen, die aus der Änderung des unübersichtlichen Gefüges der regionalen Konstellation resultierte. So tendierten die türkischen Entscheidungsträger dazu, die Entstehung und vor allem die Eskalation zwischenstaatlicher Konflikte sowie weiterer potenzieller Krisen in ihren Nachbarstaaten durch internationale Zusammenarbeit vorab oder aber zumindest rechtzeitig einzudämmen.

Eine fundamentale Neuorientierung der türkischen Außenpolitik nach dem Ende des Ost-West-Konflikts war indes nicht der Fall war, die als eine Alternative zur bisherigen pro-westlichen und pro-europäischen Außenpolitik mit dem transepochalen Ziel einer weitgehenden Integration mit den sicherheitspolitischen, institutionelllen und wirtschaftlichen Kooperationsformen mit dem Westen betrachtet werden könnte.

Bei der neuen Außenpolitik handelte es sich weniger um Machtpolitik oder das Anstreben einer Machtexpansion, sondern vielmehr darum, in den neuen polyzentrischen regionalen Konstellation anhand neuer Perzeptionen und Interessenslagen in einem kontinuierlichen Interaktionsprozess mit den anderen involvierten Akteuren eine neue Außenpolitik zu gestalten. Dies ist der Türkei im Analysenzeitraum größtenteils gelungen. Unterstrichen werden soll hierbei die Tatsache, dass die Entstehung einer neuen Regionalpolitik entsprechend den neuen Parametern der internationalen Politik nach dem Ende des Ost-West-Konflikts keine optionale Neuorientierung, sondern eine unvermeidbare Anpassung darstellt. 


\section{Zusammenfassung}

Das Ende des Ost-West-Konflikts veränderte die Rahmenbedingungen der türkischen Außenpolitik. Während die Türkei parallel zu ihrem Wertverlust im sicherheitspolitischen Kontext in Europa Schwierigkeiten hatte, ihren traditionellen Europakurs mit dem Ziel der Mitgliedschaft in der EU zu legitimieren, entstanden für die Türkei nach der Auflösung der Sowjetunion neue Chancen im Kaukasus und in Zentralasien. Dieser Artikel beabsichtigt, die türkische Außenpolitik unter diesen neuen Konditionen der internationalen Politik zu verorten. Dabei wird versucht, die Frage zu beantworten, ob es sich bei dem neuen Außenpolitikkonzept um ein außenpolitisches Potenzial als Alternative zur sich bisher als dominante Orientierung erwiesenen Politik mit dem Ziel des EU-Beitritts handelt, oder ob diese neue Außenpolitik anders kategorisiert werden kann.

\section{Summary}

The end of the Cold War has changed the general conditions of the turkish foreign policy. While Turkey had some difficulties in the post Cold War period parallel to its lower importance in european security context to legitimate its european course with the final destination of the full membership in the EU, there were new chances in Caucasus and Central Asia after the disintegration of the Soviet Union. This article intends to locate the Turkish foreign policy under these new conditions of the international relations. It tries to answer the question whether it concerns by the new foreign policy concept a potential as alternative to dominating orientation with the destination of EU membership, or whether this orientation could be categorized differently as well.

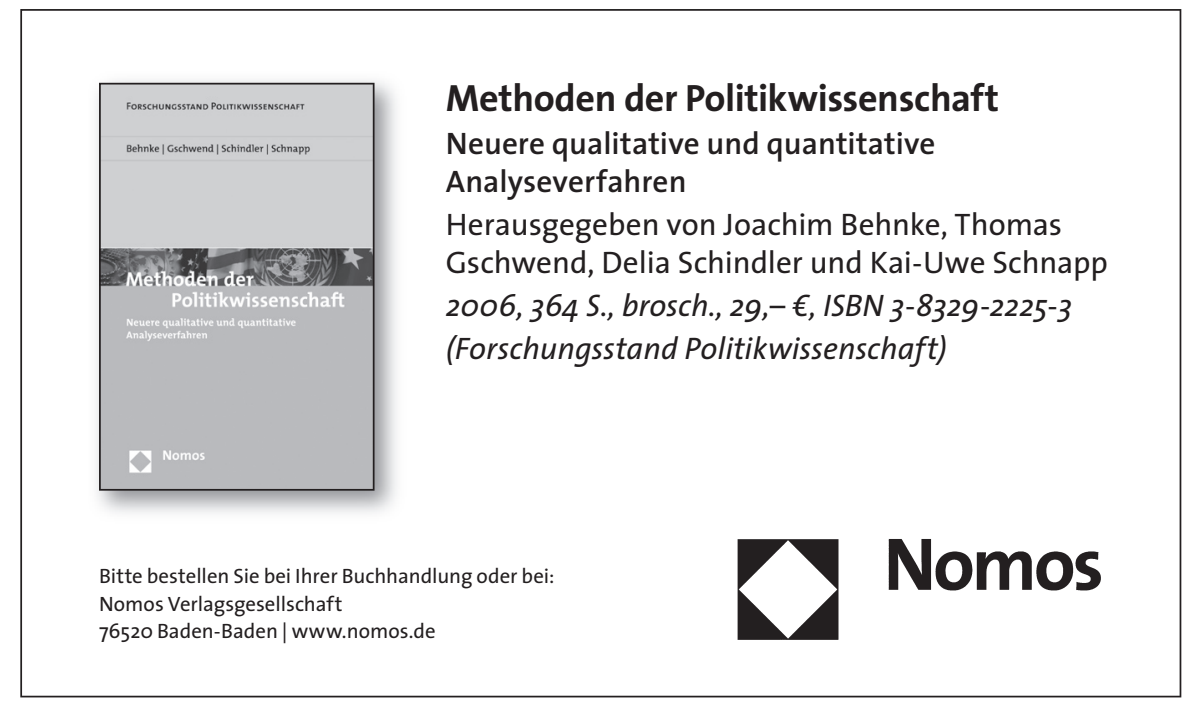

